\title{
Research on the Deformation Measurement of Ground Collapse Based on Spiral Parallel Cable and TDR
}

\author{
Bo Cao ${ }^{1,}$, , Qing $\mathrm{Li}^{2, \mathrm{~b} *}$, YangKai Zhang ${ }^{3, \mathrm{c}}$ \\ Institute of mechanical and electrical engineering \\ China Jiliang University, China \\ a592522956@qq.com, blq13306532957@163.com
}

Keywords: spiral parallel cable; TDR; ground collapse deformation

\begin{abstract}
This paper briefly describes the defects of the ground collapse measurement technology at present and proposes a new method for measuring the ground collapse. The structure of the spiral parallel cable is illustrated and its circuit characteristics are deduced,it is concluded that the characteristic impedance increases with the increase of tensile deformation, and the dielectric constant has little effect on the characteristic impedance. TDR method combined with spiral parallel cable can be applied to the measurement of ground collapse.Tensile deformation reflects the extent of ground collapse, and the position of the peak of the reflection signal is the location of the ground collapse deformation. In the end,the mathematical model of the UVE-PLS which sets up the relationship between the tensile deformation and the reflection signal is established,and gets accurate prediction results.
\end{abstract}

\section{Introduction}

Ground collapse refers to the surface subsidence or collapse due to the continued development or environmental conditions change in the subsurface minerals being mined, and It has important significance for the early warning and monitoring[1].

Although there are many methods to measure the surface subsidence currently, but most of them exist in the defect of real-time for measurement are not enough[2][3], so it needs a distributed, used for continuous monitoring,remote control, and accurate determination of the ground subsidence deformation position method. At present, the measurement method of meeting this requirement are technology of the coaxial cable based on TDR and optical fiber sensing technology(such as BOTDR, BOTDA, OTDR)[4][5], but these two methods have their own shortcomings.

The distributed measurement technique of coaxial cable is used to measure the displacement of the slope. It has the merit of excellent distributed deformation measurement,however, there are also many shortcomings at the same time: Firstly, the coaxial cable is limited by the structure of its central electric wire, so its deformation is very limited compared to the deformation of the geological hazard.Secondly, the deformation of the coaxial cable is confined to the shear deformation of the cable cross section, and can not be used for the distributed measurement of the deformation of surface tension, subsidence or uplift. The reliability and stability of the sensing technology based on optical fiber is important in the field of building engineering testing, but it has a fatal weakness: The deformation of the optical fiber is very limited which may be cracked caused by large deformation such as ground subsidence.

So this paper proposes a novel and reliable method that can be used to meet the distributed measurement and can overcome the sensors defects of the coaxial cable and fiber. The method is measuring the deformation of ground collapse based on spiral parallel cable and TDR.

\section{The Structure and Circuit Model of Spiral Parallel Cable}

The Structure of Spiral Parallel Cable. In this paper, the structure of the spiral parallel cable is designed based on the structure of the coaxial cable.As shown in figure 1, from the inside to the outside, the first layer and the second layer of copper wires are evenly around the cylindrical elastic 
silica gel surface,and ensured that copper wires keep parallel to each other.Finally, sealing the external copper wires with the silica gel, which plays a waterproof and insulation role.

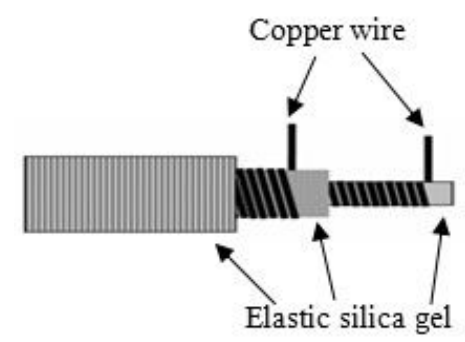

Figure 1. The structure of spiral parallel cable

The production of the spiral parallel cable in kind is as figure 2 and its total diameter is $7.57 \mathrm{~mm}$.Compared with the coaxial cable, spiral parallel cable has greater tensile deformation, and copper wires in the tensile process do not break. Therefore, spiral parallel cable this paper proposed is more suitable for the measurement of surface subsidence deformation.

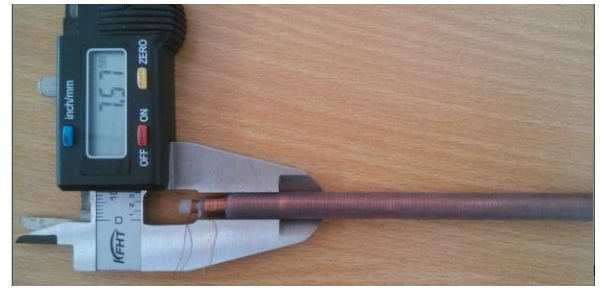

Figure 2. Picture of spiral parallel cable

The Uniform Transmission Cable Model. Uniform transmission cable refers to the two conductor parallel to each other in the medium. The spiral parallel cable referred in this paper meets the uniform transmission cable definition, so the circuit model of the uniform transmission cable can be used to analyze the circuit characteristics of the spiral parallel cable.

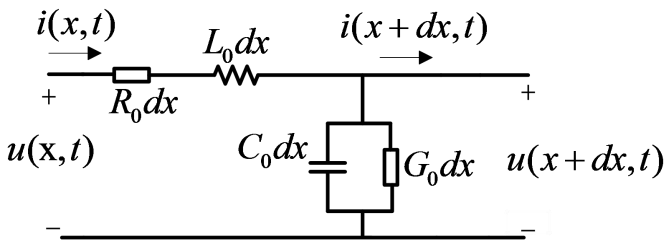

Figure 3. Equivalent circuit of uniform transmission cable

In figure $3, R_{0}$ is unit length resistance, $L_{0}$ is unit length inductance, $C_{0}$ is unit length capacitance and $G_{0}$ is unit length conductance. The characteristic impedance of a uniform transmission cable can be represented by the formula (1).

$$
Z_{C}=\sqrt{\left(R_{0}+j w L_{0}\right) /\left(\mathrm{G}_{0}+j w C_{0}\right)}
$$

When $R_{0}<<L_{0}$ and $G_{0}<<C_{0}$, characteristic impedance can be reduced to formula (2).

$$
Z_{C}=\sqrt{L_{0} / C_{0}}
$$

Characteristic Impedance of Spiral Parallel Cable. In this section,the spiral parallel cable distributed capacitance $C_{0}$ and distributed inductance $L_{0}$ are derived. When the parallel cable is not stretched, the copper wires of each layer are closely connected, which can be regarded as a cylindrical capacitor[7]. When the spiral parallel cable is stretched, the deformation occurs as shown in figure 4(take second layers of dense copper wire for example), and assuming the tensile length is $d$.So the distribution of the spiral parallel cable is made up of two parts. A part is not stretched when the distribution of the $C_{A}$, the other part is stretched when the distribution of capacitance $C_{B}$, that is to say $C_{0}=C_{A}+C_{B}$.

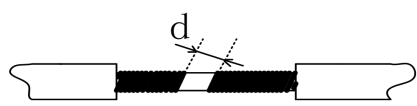

Figure 4. Picture of spiral parallel cable stretched 
The derivation of the distribution capacitance of the cylindrical capacitor according to the electromagnetic field[6] as follows:

$$
C_{A}=2 \pi \varepsilon \ln \left(R_{A} / R_{B}\right)
$$

In formula (3), $\varepsilon$ is silica gel dielectric constant, $R_{A}$ is the radius of the first layer of silica gel. $R_{B}$ is the radius of the first layer of silica gel.Analyzing the distribution capacitance of the spiral parallel cable drawn, the tensile part of the copper wire can be regarded as a parallel straight wire model. Suppose that the stretching length of the spiral parallel cable is $d$, and copper wire radius is $r$, then the distribution capacitance of the parallel wire drawing is satisfied with the following formula:

$$
C_{B}=2 \pi \varepsilon \ln (r /(d-r))
$$

So the distribution of the spiral parallel cable $C_{0}$ meets the following formula:

$$
C_{0}=C_{A}+C_{B}=2 \pi \varepsilon \ln \left(\frac{r R_{A}}{d R_{B}}\right)
$$

When the signal frequency is high enough, the charge will focus on the surface, then the electromagnetic wave will be all located in the medium of the helix. So the distribution capacitance and inductance are as follows, and $v$ is electromagnetic wave velocity.

$$
v=1 / \sqrt{L_{0} C_{0}}=1 / \sqrt{\varepsilon \mu}
$$

So the formula of the characteristic impedance of the helix parallel line can be deduced as follows:

$$
Z_{C}=1 /(2 \pi) \sqrt{\mu / \varepsilon} \ln \left(d R_{B} /\left(r R_{A}\right)\right)
$$

In formula(7), $\mu$ is permeability of the medium, $\varepsilon$ is dielectric constant, $d$ is stretching distance, $r$ is copper wire radius, $R_{B}$ is second layer of silica gel, and $R_{A}$ is first layer of silica gel.

Table1. Parameter Values

\begin{tabular}{cccc}
\hline$\mu$ & $r$ & $R_{A}$ & $R_{B}$ \\
\hline \hline 1 & $0.2 \mathrm{~mm}$ & $1.5 \mathrm{~mm}$ & $3 \mathrm{~mm}$
\end{tabular}

Putting the parameters values shown in table 1 into the formula (7), then relationship of the characteristic impedance,dielectric constant and the stretching amount is shown in figure 5.As shown in figure 5, the dielectric constant has little influence on the characteristic impedance of the spiral parallel cable,and the characteristic impedance and dielectric constant are monotonically decreasing,besides, the impedance of the spiral parallel cable increases with the increase of the stretching quantity.

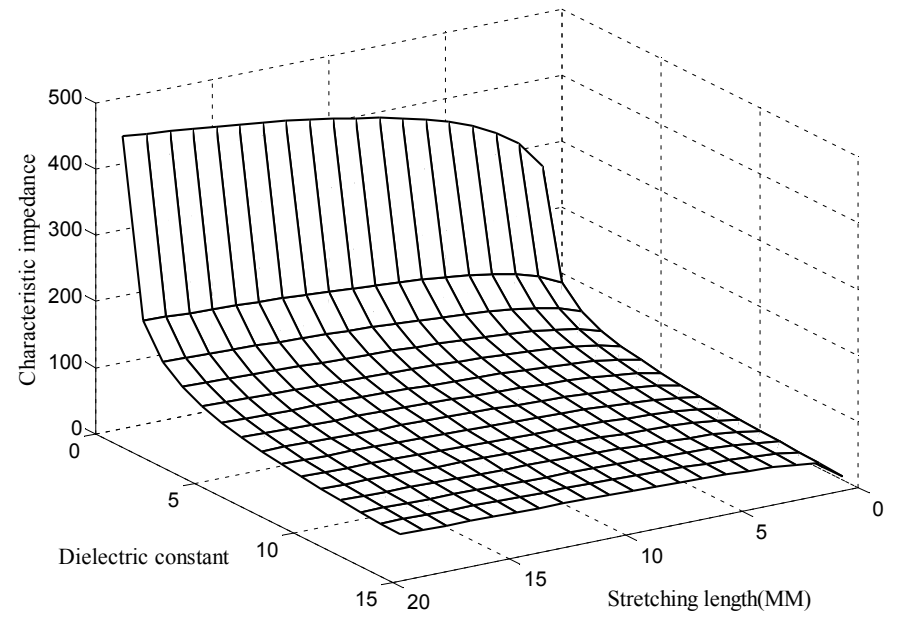

Figure 5. Relationship of characteristic impedance, dielectric constant and tension

\section{Study on Time Domain Reflection of Spiral Parallel Cable}

TDR is short for time domain reflection. Time domain reflective measuring instrument generates an excitation signal input to the spiral parallel cable,then the impedance of the deformation will change when the spiral parallel cable is stretched, and the excitation signal in the location of the 
impedance changed will produce a reflection signal.By studying the amplitude of the reflected signal, stretching position and stretching length, we can measure the position and collapse degree of the surface subsidence deformation. The TDR measurement of the spiral parallel cable is shown in figure 6.

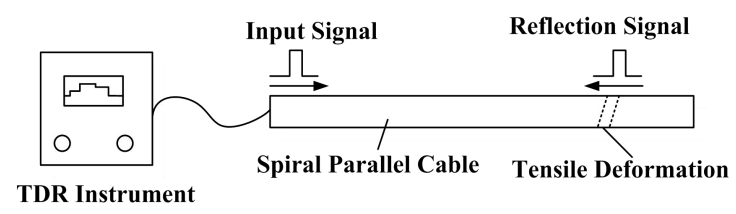

Figure 6. Time domain reflection principle

In this paper, a $100 \mathrm{~cm}$ long spiral parallel cable which there are 60 meters copper wire inside is spread on the experimental device that is shown in figure 7.Shorting circuit of two copper wire at the end of the cable,and pressing it by plates. The distance between pressed position and end of cable is $25 \mathrm{~cm}$ which is 15 meters away between copper wire pressed position and the end of copper wire.

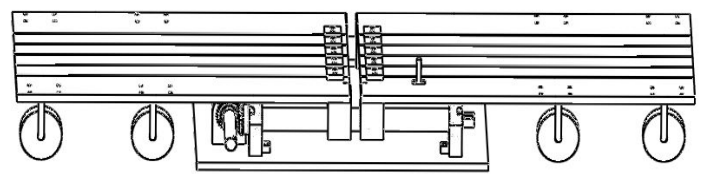

Figure 7. Structure of experimental device

Rotating the drive shaft of the experimental device shown in figure 7,then the plate is stretched by experimental device's internal gear,and finally it achieves the purpose of drawing spiral parallel cable.In this paper, the TDR module of Tektronix DSA8300 Digital Serial Analyzer is used to transmit step signal and receive the reflected signal waveform, and vernier caliper fixed on the plate is used to measure the tensile capacity when the spiral parallel cable is stretched.The whole experimental environment is shown in figure 8.

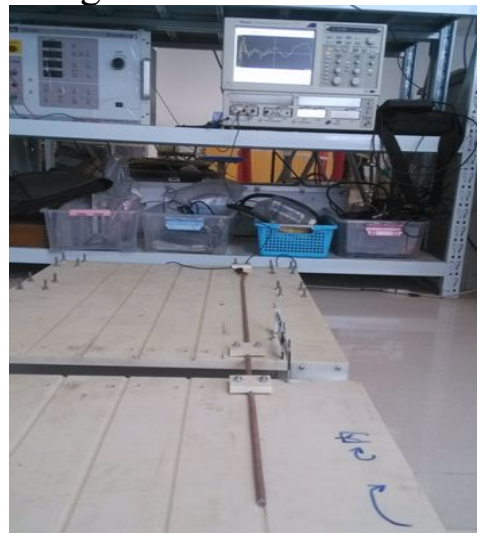

Figure 8. Experimental photo

The spiral parallel cable is stretched from $1 \mathrm{~cm}$ to $6 \mathrm{~cm}$ in the same position,and the relationship of reflected signal amplitude,the stretching position and the stretching length are obtained after experimenting as shown in figure 9.

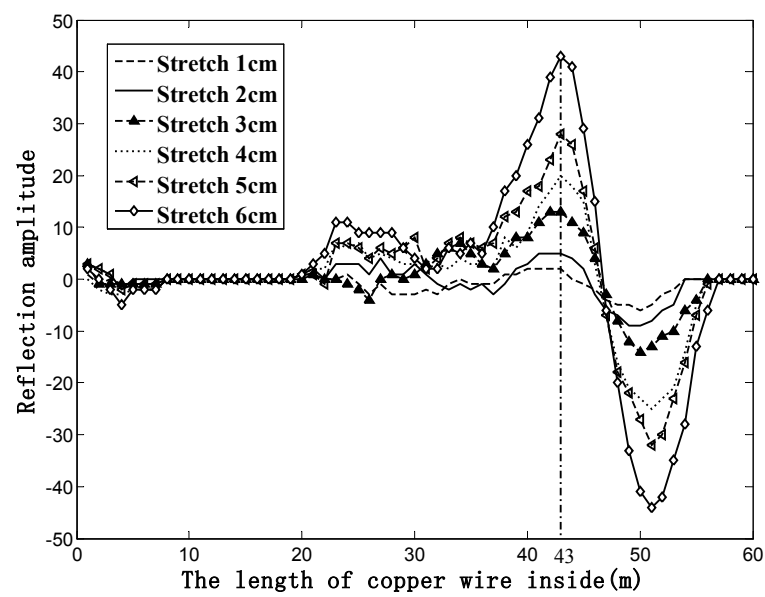

Figure 9. Relation of reflection amplitude,tensile position and tension length 
Because the signal transmitting and receiving relate to copper wire, so abscissa in figure 9 is expressed by the length of copper wire in the spiral parallel cable,that is to say, the abscissa measuring range is $60 \mathrm{~m}$.From figure 9 , the greater tensile capacity of the spiral cable is, the larger reflection signal amplitude of the TDR instrument is,and the maximum amplitude of the corresponding horizontal coordinates is the same that expresses the deformation position is the same.In theory, the deformation position should be in the horizontal coordinates of 45 while the calculated value is 43 ,and this is due to the reason that the copper wire length has been neglected under the two pressure plates.

\section{Establishment of UVE-PLS Model and Prediction of Surface Subsidence}

According to the experimental results of the third section, it is obviously known that the spiral parallel cable can be used to measure the ground collapse completely.Because the ground internal deformation will occur before ground collapsing that results in the deformation of the spiral parallel cable, so combined with the analysis of the reflection signal the TDR instrument received we can determine the extent degree of ground collapse. The position of the maximum reflection signal value expresses the position of the collapse deformation, and the stretch length of the parallel cable expressed the degree of the ground collapse.In this paper, 59 sets of experiments are done on the different stretching lengths of the spiral parallel cable in different positions,and 2000 data of reflective signals are acquired in each group experiment.Based on the UVE-PLS model, the relationship between the stretching length of the spiral parallel cable and the data of the reflected signal can be established, finally we can use the model to predict the of the ground subsidence deformation.

UVE is a new variable selection method based on the stability analysis of regression coefficients which can effectively eliminate the useless information variables and prevent the prediction model over fitting,and its detailed algorithm and principle can be consulted in literature[7].PLS is the abbreviation of partial least square method that is a new analysis method of multivariate statistical data.Because each group of experimental data is too much, so the UVE algorithm is used to extract 1000 valid data from each group,and we use the PLS algorithm to carry on the multiple linear fitting with the 49 set of experimental data,in the end, the rest 10 sets of experimental data are used to predicted.The main purpose of PLS is to build a linear model as following:

$$
\left[\begin{array}{l}
y_{1} \\
y_{2} \\
\vdots \\
y_{49}
\end{array}\right]=\left[\begin{array}{cccc}
x_{1,1} & x_{1,2} & \cdots & x_{1,1000} \\
x_{2,1} & x_{2,2} & \cdots & x_{2,1000} \\
\vdots & \vdots & \ddots & \vdots \\
x_{49,1} & x_{49,2} & \cdots & x_{49,1000}
\end{array}\right]\left[\begin{array}{l}
k_{1} \\
k_{2} \\
\vdots \\
k_{1000}
\end{array}\right]+b
$$

In formula (8), $y_{n}$ represents the length of the $n$ test cable stretching, $x_{n, m}$ means that the reflection signal of the $n$ experiment obtained from the $m$ data value, $k_{m}$ represents the coefficient of the $m$ data values, and $b$ is a constant.

Taking the experimental data into the above formula,we obtain $k_{m}$ values and $b=3725.2$. The 10 reset sets of experimental data not be involved in the calculation of the PLS used to predictive validation, and the predicted results of tensile length and deformation position are shown in Table 2 and table 3 .

\begin{tabular}{c|cccc}
\multicolumn{5}{c}{ Table2. Prediction of tensile length } \\
Actual & 6.5 & 12.5 & 18.5 & 24.5 \\
stretch & 8.5 & 14.5 & 20.5 & \\
length/cm & 10.5 & 16.5 & 22.5 & \\
\hline \hline & & & & \\
Predicted & 6.176 & 12.241 & 18.350 & 24.548 \\
stretch & 8.583 & 14.742 & 20.551 & \\
length/cm & 10.406 & 16.781 & 22.662 & \\
\hline
\end{tabular}

\begin{tabular}{c|cccc}
\multicolumn{5}{c}{ Table3. Prediction of tensile position } \\
\hline $\begin{array}{c}\text { Actual } \\
\text { deformation }\end{array}$ & 50 & 75 & 65 & 65 \\
position/cm & 50 & 65 & 40 & \\
\hline \hline & 18.7 & 74.7 & 63.2 & 63.8 \\
Predicted & & & & \\
deformation & 49.2 & 68.8 & 44.4 & \\
position/cm & 49.3 & 63.7 & 38.8 & \\
\hline
\end{tabular}




\section{Conclusions}

In this paper, the method of time domain reflection measurement based on the spiral parallel cable is demonstrated to be uesd in distributed measurement of surface subsidence deformation field in two aspects: the analysis of the circuit characteristics and the experimental results,and spiral parallel cable lays the foundation for the actual application of ground subsidence.

\section{Acknowledgment}

This work is funded by the National Natural Science Foundation of China(NSFC) under Grant No.60772008, project of the NSFC under Grant No.41376111, and the National Science and Technology Support Plan of China under Grant No.2012BAK20B05-3.

\section{References}

[1] Yu Dapeng, Qun Qiuya, Ma Bingzhi, Mao Wei. Risk Prediction of Ground Subsidence in Mined-out Area[J]. Industry and Mine Automation, 2010, 03:78-81.

[2] Guo Guangli, Miao Xiexing, Zhang Zhennan. Research on Ruptured Rock Mass Deformation Characteristics of Longwall Goafs[J]. Science Technology and Engineering, 2002, 05:44-47.

[3] Wang Jianliang, Yao Ji, Pu Xiurong. Analyze aweak rock Slope's Stability with FLAC \&Limit Equilibrium Method[J]. Science Technology and Engineering, 2009, 16:4693-4697.

[4] Jiang Xiaozhen, Lei Mingtang, Gu Weifang,Zhang Meiheng. Soil cave monitoring and sinkhole prediction in linear engineering:summarize[J]. Carsologica Sinica, 2008, 02:172-176.

[5] Jiang Xiaozhen, Lei Mingtang, Gu Weifang,Zhang Meiheng. Soil cave monitoring and sinkhole prediction in linear engineering:summarize[J]. Carsologica Sinica, 2008, 02:172-176.

[6] Tong Renyuan. Spiral Cable-based Geotechnical Deformation Distributed Measurement Technology Research[D]. East China Normal University, 2013

[7] Zhong Rupeng, Wang Yuxi, Xu Fuyuan, Xiao Zuobing. Cigarette smoke component analysis for the taste characteristics by using UVE-SPA[J]. Science-Technology and Management, 2011, 02:40-43. 